\title{
SCIENTIFIC REPORTS

\section{Diagnostic performance of axillary ultrasound and standard breast MRI for differentiation between limited and advanced axillary nodal disease in clinically node-positive breast cancer patients}

S. Samiei ${ }^{1,2,3^{*}}$, T. J. A. van Nijnatten ${ }^{2}$, H. C. van Beek ${ }^{4}$, M. P. J. Polak ${ }^{4}$, A. J. G. Maaskant-Braat ${ }^{5}$, E. M. Heuts ${ }^{1}$, S. M. J. van Kuijk ${ }^{6}$, R. J. Schipper ${ }^{1}$, M. B. I. Lobbes ${ }^{2,3,7}$ \& M. L. Smidtt ${ }^{1,3,7}$

Preoperative differentiation between limited ( $\mathrm{pN1} ; 1-3$ axillary metastases) and advanced ( $\mathrm{pN2}-3$; $\geq 4$ axillary metastases) nodal disease can provide relevant information regarding surgical planning and guiding adjuvant radiation therapy. The aim was to evaluate the diagnostic performance of preoperative axillary ultrasound (US) and breast MRI for differentiation between pN1 and pN2-3 in clinically node-positive breast cancer. A total of 49 patients were included with axillary metastasis confirmed by US-guided tissue sampling. All had undergone breast MRI between 2008-2014 and subsequent axillary lymph node dissection. Unenhanced T2-weighted MRI exams were reviewed by two radiologists independently. Each lymph node on the MRI exams was scored using a confidence scale (0-4) and compared with histopathology. Diagnostic performance parameters were calculated for differentiation between pN1 and pN2-3. Interobserver agreement was determined using Cohen's kappa coefficient. At final histopathology, 67.3\% (33/49) and 32.7\% (16/49) of patients were pN1 and pN2-3, respectively. Breast MRI was comparable to US in terms of accuracy (MRI reader 1 vs US, $71.4 \%$ vs $69.4 \%, p=0.99$; MRI reader 2 vs US, $73.5 \%$ vs $69.4 \%, p=0.77$ ). In the case of $1-3$ suspicious lymph nodes, pN2-3 was observed in $30.4 \%$ on US (positive predictive value (PPV) $69.6 \%$ ) and in $22.2-24.3 \%$ on MRI (PPV 75.7-77.8\%). In the case of $\geq 4$ suspicious lymph nodes, pN1 was observed in $33.3 \%$ on US (negative predictive value (NPV) 66.7\%) and in $38.5-41.7 \%$ on MRI (NPV 58.3-61.5\%). Interobserver agreement was considered good $(k=0.73)$. In clinically node-positive patients, the diagnostic performance of axillary US and breast MRI is comparable and limited for accurate differentiation between $\mathrm{pN} 1$ and $\mathrm{pN2}-3$. Therefore, there seems no added clinical value of preoperative breast MRI regarding nodal staging in patients with positive axillary US.

The axillary lymph node status in breast cancer patients provides relevant information regarding treatment planning and prognosis ${ }^{1,2}$. In our current breast cancer workup, a preoperative axillary ultrasound (US) is routinely performed for evaluation of the axillary lymph nodes ${ }^{3-5}$. If suspicious axillary lymph nodes are detected with US, fine needle aspiration cytology (FNAC) or core needle biopsy is performed to identify clinically node-positive

\footnotetext{
${ }^{1}$ Department of Surgery, Maastricht University Medical Centre+, Maastricht, The Netherlands. ${ }^{2}$ Department of Radiology and Nuclear Medicine, Maastricht University Medical Centre+, Maastricht, The Netherlands. ${ }^{3}$ GROW - School for Oncology and Developmental Biology, Maastricht University Medical Centre+, Maastricht, The Netherlands. ${ }^{4}$ Department of Radiology, Maxima Medical Centre, Eindhoven, The Netherlands. ${ }^{5}$ Department of Surgery, Maxima Medical Centre, Eindhoven, The Netherlands. ${ }^{6}$ Department of Clinical Epidemiology and Medical Technology Assessment, Maastricht University Medical Centre+, Maastricht, The Netherlands. ${ }^{7}$ These authors jointly supervised this work: M. B. I. Lobbes and M. L. Smidt. *email: sanaz.samiei@mumc.nl
} 
patients. Formerly, an axillary lymph node dissection (ALND) was performed in these clinically node-positive patients as standard practice. With increasing use of neoadjuvant systemic therapy (NST) and therefore the advantage of possible downstaging of the axilla, alternative approaches are proposed for the axillary lymph node staging, such as sentinel lymph node biopsy (SLNB), marking axillary lymph nodes with radioactive iodine seed (MARI), targeted axillary dissection (TAD), and combining radioactive iodine seed localisation in the axilla with the sentinel node procedure (RISAS), all to avoid ALND associated comorbidities ${ }^{6-9}$. By using such alternative approaches, the true pathological axillary lymph node status is unknown since these techniques have false-negative findings of up to $15 \% \%^{6,7,10}$. Combining an imaging technique before NST with these less invasive surgical staging methods, such as SLNB, MARI, TAD, or RISAS, can contribute to the selection of patients for whom further axillary treatment is (un)necessary.

In clinically node-negative patients treated with primary surgery, the role of a complementary ALND in sentinel node-positive breast cancer patients has been addressed in previous trials, such as the ACOSOG Z0011 and AMAROS $^{11,12}$. These trials showed that omitting ALND or in the case of treatment with axillary radiotherapy in sentinel node-positive patients did not result in worse locoregional recurrence or survival rate ${ }^{11-13}$. When omitting (complementary) ALND or in the case of treatment with axillary radiotherapy, some patients with advanced axillary nodal disease (pN2-3; i.e., $\geq 4$ axillary lymph node metastases) might not be identified. Patients with advanced axillary nodal disease are recommended to undergo radiation therapy of the chest wall or (reconstructed) breast with the addition of infraclavicular/supraclavicular lymph nodes, an imaging technique to identify pN2-3 disease before surgery is necessary.

Consequently, preoperative differentiation between limited ( $\mathrm{pN} 1$; i.e., 1-3 axillary lymph node metastases) and advanced axillary nodal disease in breast cancer patients can provide relevant information regarding the surgical planning and guiding adjuvant radiation therapy. The role of breast MRI for evaluating axillary lymph node metastases has been described in earlier studies with reported sensitivity and specificity between $33.3-97.0 \%$ and $14.0-98.5 \%$, respectively ${ }^{14-16}$. Hyun et al. showed that a negative breast MRI can exclude pN2-3 with negative predictive value (NPV) of $99.6 \%$. However, the differentiation between $\mathrm{pN} 1$ and $\mathrm{pN} 2-3$ has not been addressed earlier in clinically node-positive patients and appears to be of clinical relevance.

Therefore, this study aimed to evaluate the diagnostic performance of preoperative axillary US and standard breast MRI for differentiation between $\mathrm{pN} 1$ and $\mathrm{pN} 2-3$ in clinically node-positive breast cancer patients. Furthermore, the second aim of this study was to evaluate whether an additional preoperative standard breast MRI is of added clinical value in patients with 1-3 suspicious axillary lymph nodes on US for differentiation between $\mathrm{pN} 1$ and $\mathrm{pN} 2-3$.

\section{Material and Methods}

Study population. All patients diagnosed with invasive breast cancer between January 2008 and December 2014, who had undergone preoperative axillary US and standard breast MRI, were retrospectively identified from two medical centres (Maxima Medical Centre (MMC) and Maastricht University Medical Centre+ $(\mathrm{MUMC}+)$ ). Patients with preoperative axillary lymph node metastasis, confirmed by US-guided FNAC or core needle biopsy (i.e., clinically node-positive patients), were included for analyses. All patients had undergone primary breast surgery and ALND. Data on the patient, tumour, and treatment characteristics were retrospectively collected. The medical ethics committee of MMC and MUMC + approved this study and waived the necessity to acquire informed consent due to the retrospective study design.

Ultrasound evaluation. The preoperative axillary lymph node status was determined by the axillary US performed by dedicated breast radiologists. Different US systems were used for the axillary US examinations: Aplio XG (Toshiba Medical Systems Europe, Zoetermeer, the Netherlands) with a linear $12 \mathrm{MHz}$ array transducer, ATL-HDI5000 (Philips Healthcare, Best, the Netherlands) with a linear 5-12 MHz array transducer, and iU22-xMATRIX (Philips Healthcare, Best, the Netherlands) with a linear 2-17 MHz array transducer. During the axillary US examination, the patient was positioned with the ipsilateral hand placed behind the head if possible. The axillary region was examined in a standardised approach, starting at the low axilla (level I: inferior and lateral to the pectoralis minor muscle) and continuing upwards toward mid-axilla (level II: medial and lateral to the pectoralis minor muscle and interpectoral). The apical axilla (level III: superior and medial to the pectoralis minor muscle with apical lymph nodes) was only evaluated when suspicious axillary lymph nodes were found in level I and/or level II. In the case of suspicious axillary lymph node(s), FNAC or a 16-18 gauge core needle biopsy was performed of the most suspicious lymph node. The total number of suspicious lymph nodes was estimated and reported. Characteristics of suspicious axillary lymph nodes on US included round or irregular shape, cortical thickness greater than $2.3 \mathrm{~mm}$, diffuse cortical thickening, effacement or replacement of fatty hilum, and increased peripheral blood flow ${ }^{17-21}$.

Breast MRI acquisition and analysis. All included patients had undergone a standard breast MRI in the prone position using a 1.5 Tesla MRI scanner (Philips Healthcare, Best, the Netherlands). In MMC, they started using the Philips Gyroscan NT scanner in 2008 with a body coil which was later replaced with an 8-channel breast coil. In 2013, the MRI scanner was replaced by Philips Ingenia R4.2 scanner with a 16-channel breast coil. In MUMC+, two types of MRI scanners, Philips Ingenia and Intera, were used with a body coil that was replaced with a 16-channel breast coil in 2011. Overview of MR protocols can be found as Supplementary Material A online.

The breast MRI exams were pre-screened by a radiology resident (T.J.A.v.N.) with three years of breast imaging experience. Screening of the MRI exams for eligibility was based on the complete field of view (FOV) of the axillary region, the absence of movement artifacts, and adequate signal-to-noise ratio. Two breast radiologists with 7 years (H.C.v.B. [reader 1]) and 14 years (M.P.J.P. [reader 2]) of experience with breast MRI retrospectively 


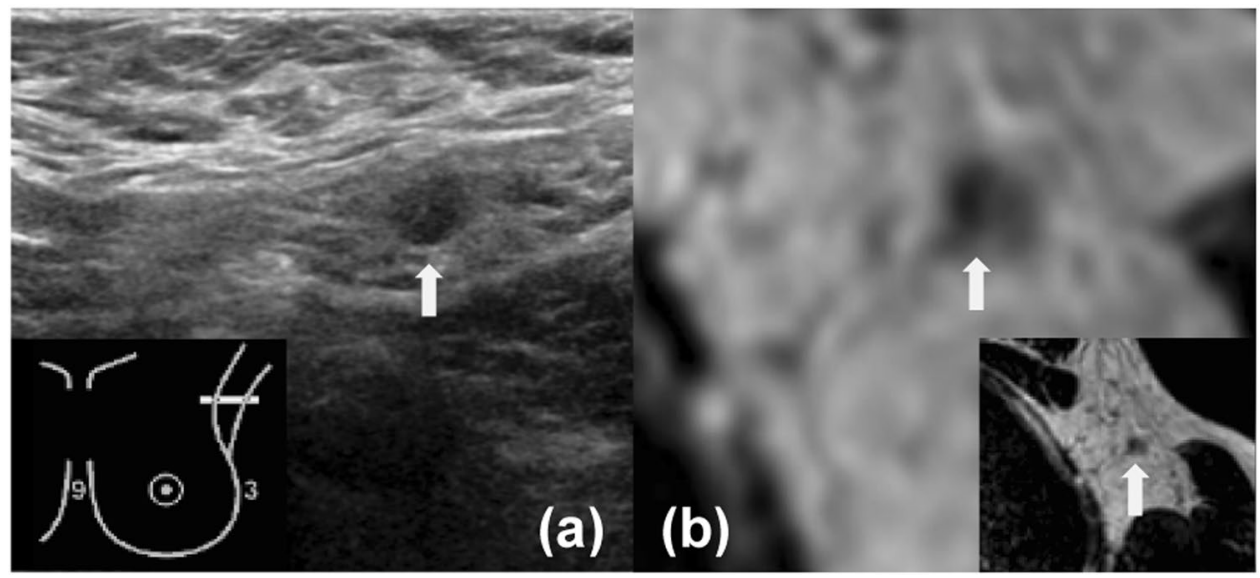

Figure 1. Images of the axilla of a 52 -year-old female patient with a $34 \mathrm{~mm}$ large invasive ductal carcinoma in her left breast, which was treated with mastectomy and ALND. For both US and MRI (reader 1 and 2) N1 axillary lymph node disease was reported. The white arrow indicates the suspicious lymph node on US and MRI. Histopathology of the ALND reported pN2-3 (largest diameter, $14 \mathrm{~mm}$ ). (a) Axillary US (b) Transversal unenhanced T2-weighted breast MRI.

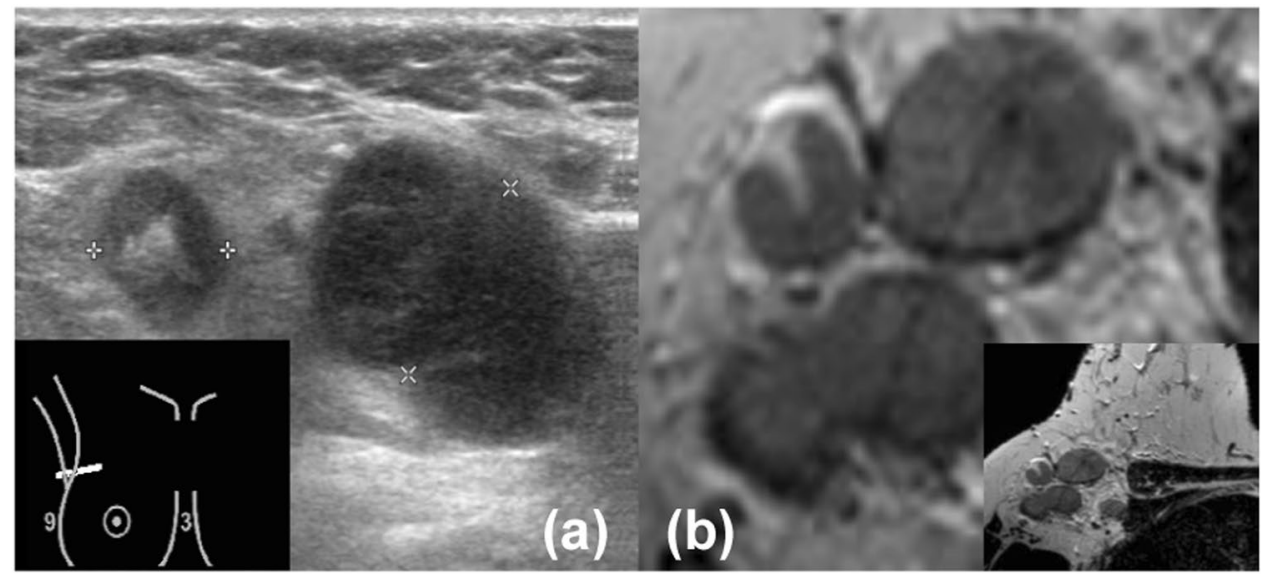

Figure 2. Images of the axilla of a 55 -year-old female patient with a $31 \mathrm{~mm}$ large invasive lobular carcinoma in her right breast, which was treated with mastectomy and ALND. For both US and MRI (reader 1 and 2) N2-3 axillary lymph node disease was reported. Histopathology of the ALND reported pN2-3 (largest diameter, $50 \mathrm{~mm}$ ). (a) Axillary US (b) Transversal unenhanced T2-weighted breast MRI.

evaluated all axillary lymph nodes (i.e., axillary level 1-3) on the unenhanced T2-weighted MR sequence. Each lymph node on the MRI exams was scored using a confidence scale ( 0 , no axillary lymph nodes; 1 , definitely benign; 2, probably benign; 3, probably malignant; 4, definitely malignant) (Figs. 1 and 2) 22 $^{2}$ Characteristics of suspicious axillary lymph nodes on MRI included irregular margins, inhomogeneous cortex, perifocal oedema, asymmetry, and absence of fatty hilum or chemical shift artifact ${ }^{22,23}$. These criteria were used to classify an axillary lymph node as either negative or positive. Similar to clinical practice, the radiologists were aware of the tumour side, clinical tumour size as assessed on MRI, and the clinical axillary lymph node status. However, they were blinded from each other's results and had no information about the final pathological axillary lymph node status.

Clinical and pathological axillary lymph node status. The clinical nodal status was based on the total number of suspicious axillary lymph nodes (N1: 1-3 suspicious lymph nodes; N2-3: $\geq 4$ suspicious lymph nodes). The ALND lymph nodes (i.e., pathological axillary lymph nodes) were paraffin-embedded after formalin fixation for histological assessment. All harvested lymph nodes with a diameter larger than $5 \mathrm{~mm}$ were sliced with a maximum thickness of $3 \mathrm{~mm}$. All slides were stained with hematoxylin and eosin. Isolated tumour cells $(\leq 0.2 \mathrm{~mm}$ and/or $<200$ cells in a single histological cross-section) and micrometastases $(0.2 \leq 2.0 \mathrm{~mm})$ were considered as negative, and macrometastases $(>2.0 \mathrm{~mm})$ as positive axillary lymph nodes. The number of axillary lymph node metastases on final histopathology defined the pathological axillary lymph node status. Pathological N1 disease was defined by 1-3 axillary lymph node metastases and pathological N2-3 disease was defined by $\geq 4$ axillary lymph node metastases ${ }^{24}$. 


\begin{tabular}{|c|c|}
\hline & All patients $(n=49)$ \\
\hline Age (years) (mean; range) & 57 [34-79] \\
\hline Clinical tumour size $(\mathrm{mm})$ (mean; range) & $35[4-100]$ \\
\hline \multicolumn{2}{|l|}{ Clinical tumour stage (\%) } \\
\hline $\mathrm{T} 1$ & $9(18.4)$ \\
\hline $\mathrm{T} 2$ & $31(63.2)$ \\
\hline T3 & $9(18.4)$ \\
\hline \multicolumn{2}{|l|}{ Breast surgery (\%) } \\
\hline Breast-conserving surgery & $8(16.3)$ \\
\hline Mastectomy & $41(83.7)$ \\
\hline \multicolumn{2}{|l|}{ Pathological nodal stage (\%) } \\
\hline $\mathrm{pN1}$ & $33(67.3)$ \\
\hline $\mathrm{pN} 2$ & $9(18.4)$ \\
\hline $\mathrm{pN} 3$ & $7(14.3)$ \\
\hline \multicolumn{2}{|l|}{ Tumour histology (\%) } \\
\hline Ductal & $40(81.6)$ \\
\hline Lobular & $8(16.3)$ \\
\hline Mixed ductal and lobular & $1(2.1)$ \\
\hline \multicolumn{2}{|l|}{ Uni - or multifocal tumour (\%) } \\
\hline Unifocal & $36(73.5)$ \\
\hline Multifocal and/or multicentric & $13(26.5)$ \\
\hline \multicolumn{2}{|l|}{ Tumour grade (\%) } \\
\hline 1 & $4(8.2)$ \\
\hline 2 & $26(53.1)$ \\
\hline 3 & $19(38.7)$ \\
\hline \multicolumn{2}{|l|}{ Receptor status (\%) } \\
\hline ER + HER2+ & $3(6.1)$ \\
\hline ER + HER2- & $33(67.4)$ \\
\hline ER-HER2 + & $6(12.2)$ \\
\hline Triple negative & $7(14.3)$ \\
\hline
\end{tabular}

Table 1. Patient, tumour, and treatment characteristics. Abbreviations: ER, Estrogen Receptor; HER2, Human Epidermal growth factor Receptor 2; Triple negative, negative for ER, PR, and HER2.

Statistical analysis. The confidence scale was dichotomised into benign (lymph nodes scores 0-2) and malignant (lymph nodes scores 3-4). Histopathology served as the gold standard. Diagnostic performance parameters (sensitivity, specificity, positive predictive value (PPV), NPV, accuracy) were calculated for axillary US and breast MRI for differentiation between $\mathrm{pN} 1$ and $\mathrm{pN} 2-3$. Sensitivity and specificity were defined as probabilities that in the case of $\mathrm{pN} 1$ or $\mathrm{pN2}-3$, imaging showed 1-3 or $\geq 4$ suspicious axillary lymph nodes, respectively. PPV and NPV were defined as probabilities that in the case of $1-3$ and $\geq 4$ suspicious axillary lymph nodes on imaging, the patient truly had $\mathrm{pN} 1$ and $\mathrm{pN} 2-3$, respectively. Comparison of sensitivity, specificity, and accuracy of US and MRI were performed with the McNemar test for paired proportions. The PPV and NPV of US and MRI were compared by using the generalised score test statistics ${ }^{25}$. In the subgroup analysis, the diagnostic performance parameters of breast MRI for differentiation between $\mathrm{pN} 1$ and $\mathrm{pN} 2-3$ were calculated for patients who had 1-3 suspicious axillary lymph nodes on US. The diagnostic performance parameters were presented with $95 \%$ confidence intervals (CIs). The reported CIs and p-values were two-sided and 0.05 was used as a cutoff for significance. Interobserver agreement between the two radiologists for evaluating the breast MRI exams was calculated by using Cohen's kappa coefficient $(\mathrm{k}<0.2=$ poor, $0.21-0.40=$ fair, $0.41-0.60=$ moderate, $0.61-0.80=$ good, $0.81-1.0=$ very good $)^{26}$. Statistical analyses were performed by using R project software (version 3.5.1, R Foundation for Statistical Computing, Vienna, Austria).

\section{Results}

A total of 49 patients [mean age, 57 years; range, 34-79 years] were included for analyses; 17 patients from MMC and 32 patients from MUMC + . Mastectomy was performed in $41(83.7 \%)$ patients and breast-conserving surgery was performed in $8(16.3 \%)$ patients. The most common tumour histology and receptor subtype were of ductal origin (81.6\%) and ER + HER2- subtype (67.3\%), respectively. At final histopathology, 67.3\% (33/49) and $32.7 \%(16 / 49)$ of the patients had $\mathrm{pN} 1$ and $\mathrm{pN} 2-3$ axillary lymph node disease, respectively. The mean size of the macrometastases was $15 \mathrm{~mm}$ (range, 6-50 $\mathrm{mm}$ ). Patient, tumour, and treatment characteristics are summarised in Table 1.

Diagnostic performance of axillary ultrasound. Of the 49 patients classified as clinically node-positive on US, 46 (93.9\%) were classified as having N1 axillary lymph node disease and $3(6.1 \%)$ were classified as having $\mathrm{N} 2-3$ axillary lymph node disease. In the case of 1-3 suspicious axillary lymph nodes evaluated on US, pN2-3 


\begin{tabular}{|l|l|l|l|l|l|}
\hline & US (n=49) & $\begin{array}{l}\text { MRI reader 1 } \\
(\mathbf{n = 4 9 )}\end{array}$ & $\begin{array}{l}\text { p-value } \\
\text { vs MRI1 }^{\mathbf{a}} \text { US }\end{array}$ & $\begin{array}{l}\text { MRI reader 2 } \\
(\mathbf{n}=49)\end{array}$ & $\begin{array}{l}\mathbf{p}^{\text {-value }} \mathbf{b} \text { US } \\
\text { vs MRI2 }^{\text {M }}\end{array}$ \\
\hline Sensitivity & $\begin{array}{l}97.0 \%(32 / 33) \\
{[84.2-99.9]}\end{array}$ & $\begin{array}{l}84.8 \%(28 / 33) \\
{[68.1-94.9]}\end{array}$ & 0.10 & $\begin{array}{l}84.8 \%(28 / 33) \\
{[68.1-94.9]}\end{array}$ & 0.10 \\
\hline Specificity & $\begin{array}{l}12.5 \%(2 / 16) \\
{[1.6-38.3]}\end{array}$ & $\begin{array}{l}43.8 \%(7 / 16) \\
{[19.8-70.1]}\end{array}$ & 0.03 & $\begin{array}{l}50.0 \%(8 / 16) \\
{[24.7-75.3]}\end{array}$ & 0.01 \\
\hline PPV & $\begin{array}{l}69.6 \%(32 / 46) \\
{[54.2-82.3]}\end{array}$ & $\begin{array}{l}75.7 \%(28 / 37) \\
{[58.8-88.2]}\end{array}$ & 0.13 & $\begin{array}{l}77.8 \%(28 / 36) \\
{[60.8-89.9]}\end{array}$ & 0.06 \\
\hline NPV & $\begin{array}{l}66.7 \%(2 / 3) \\
{[9.4-99.2]}\end{array}$ & $\begin{array}{l}58.3 \%(7 / 12) \\
{[27.7-84.8]}\end{array}$ & 0.77 & $\begin{array}{l}61.5 \%(8 / 13) \\
{[31.6-86.1]}\end{array}$ & 0.85 \\
\hline Accuracy & $\begin{array}{l}69.4 \%(34 / 49) \\
{[54.6-81.7]}\end{array}$ & $\begin{array}{l}71.4 \%(35 / 49) \\
{[56.7-83.4]}\end{array}$ & 0.99 & $\begin{array}{l}73.5 \%(36 / 49) \\
{[58.9-85.1]}\end{array}$ & 0.77 \\
\hline
\end{tabular}

Table 2. Diagnostic performance of axillary US and standard breast MRI for differentiation between pN1 and pN2-3. Abbreviations: US, ultrasound; PPV, positive predictive value; NPV, negative predictive value; MRI1, MRI reader 1; MRI2, MRI reader 2. ${ }^{\mathrm{a}} \mathrm{McNemar}$ and generalised score test for comparison of diagnostic performance parameters between US and breast MRI of reader $1 .{ }^{b} \mathrm{McNemar}$ and generalised score test for comparison of diagnostic performance parameters between US and breast MRI of reader 2. Data in parenthesis are absolute numbers. Data in brackets are $95 \%$ confidence intervals.

was found in $30.4 \%$ of the patients with a PPV of $69.6 \%$. In the case of $\geq 4$ suspicious axillary lymph nodes evaluated on US, pN1 was found in $33.3 \%$ of the patients with an NPV of $66.7 \%$ (Table 2).

Diagnostic performance of standard breast MRI. Of the 49 patients that were clinically node-positive on US, reader 1 classified $37(75.5 \%)$ patients as having N1 axillary lymph node disease and $12(24.5 \%)$ patients as having N2-3 axillary lymph node disease on breast MRI. Reader 2 classified 36 (73.5\%) patients as having N1 axillary lymph node disease and $13(26.5 \%)$ patients as having N2-3 axillary lymph node disease on breast MRI. In the case of 1-3 suspicious axillary lymph nodes evaluated on breast MRI, pN2-3 was found in $22.2-24.3 \%$ of the patients with a PPV of $75.7-77.8 \%$. In the case of $\geq 4$ suspicious axillary lymph nodes evaluated on breast MRI, pN1 was found in 38.5-41.7\% of the patients with an NPV of 58.3-61.5\% (Table 2). Interobserver agreement between the two radiologists for reviewing the breast MRI exams was considered good $(\mathrm{k}=0.73)$.

Breast MRI of reader 1 was comparable to US in terms of both sensitivity ( $84.8 \%$ vs $97.0 \%, p=0.10)$, PPV (75.7\% vs $69.6 \%, \mathrm{p}=0.13)$, NPV ( $58.3 \%$ vs $66.7 \%, \mathrm{p}=0.77)$, and accuracy $(71.4 \%$ vs $69.4 \%, \mathrm{p}=0.99)$. Breast MRI of reader 1 showed better specificity $(43.8 \%$ vs $12.5 \%, \mathrm{p}=0.03)$ compared to US. Breast MRI of reader 2 was comparable to US in terms of both sensitivity $(84.8 \%$ vs $97.0 \%, \mathrm{p}=0.10)$, PPV $(77.8 \%$ vs $69.6 \%, \mathrm{p}=0.06)$, NPV $(61.5 \%$ vs $66.7 \%, \mathrm{p}=0.85)$, and accuracy $(73.5 \%$ vs $69.4 \%, \mathrm{p}=0.77)$. Breast MRI of reader 2 also showed better specificity ( $50.0 \%$ vs $12.5 \%, \mathrm{p}=0.01)$ compared to US.

The subgroup analysis showed that 46 patients had N1 axillary lymph node disease on US. Reader 1 correctly classified $75.0 \%(27 / 36)$ of the patients as having pN1 axillary lymph node disease and 50.0\% (5/10) of the patients as having pN2-3 axillary lymph node disease on breast MRI. Reader 2 correctly classified 77.1\% (27/35) of the patients as having pN1 axillary lymph node disease and $54.5 \%(6 / 11)$ of the patients as having pN2-3 axillary lymph node disease on breast MRI (Table 3). Interobserver agreement between the two radiologists for reviewing the subgroup breast MRI exams was considered good $(\mathrm{k}=0.69)$.

\section{Discussion}

Non-invasive preoperative differentiation between $\mathrm{pN} 1$ and $\mathrm{pN} 2-3$ has become increasingly important since this can provide relevant information regarding surgical planning and guiding adjuvant radiation therapy. In this study, we showed that the diagnostic performance of preoperative axillary US and standard breast MRI is comparable and inaccurate for differentiation between $\mathrm{pN} 1$ and $\mathrm{pN} 2-3$ in clinically node-positive breast cancer patients. Furthermore, when US showed 1-3 suspicious axillary lymph nodes, an additional preoperative standard breast MRI correctly diagnosed $\mathrm{pN} 2-3$ in only $50.0-54.5 \%$ of these patients.

ALND and radiation therapy to the chest wall or (reconstructed) breast and infraclavicular/supraclavicular lymph nodes are recommended in breast cancer patients with $\geq 4$ axillary lymph node metastases who are at significant risk of locoregional recurrence in the chest wall or conserved breast and nodal basins, regardless of the response to NST ${ }^{27-29}$. The number of positive nodes is not only important for guiding adjuvant radiation therapy, but also the timing of breast reconstruction in patients treated with primary surgery. Previous research has demonstrated that radiation therapy on the reconstructed breast can increase complication rates and may compromise the aesthetic outcomes, especially in implant-based reconstructions ${ }^{30-33}$. Therefore, preoperative identification of $\mathrm{pN} 2-3$ disease is preferable in patients with a desire for immediate breast reconstruction to avoid potential radiation therapy complications.

In the diagnostic workup of breast cancer patients, the US and US-guided FNAC or core needle biopsy are widely accepted for the assessment of axillary lymph node metastases given the low morbidity, cost-effectiveness, and high accuracy ${ }^{17,34}$. Previous studies have addressed the ability of axillary US for differentiation between pN0-N1 and pN2-N3 $3^{17,35}$. They reported that a negative axillary US can exclude pN2-3 with an NPV of 96.0$97.7 \%{ }^{17,35}$. In the case of a positive axillary US, the ability to exclude pN2-3 was considered insufficient with a reported NPV of $58.5-71 \%^{35,36}$. This is in line with our results. We demonstrated that the ability to exclude pN2-3 was $69.6 \%$ in the case of $1-3$ suspicious axillary lymph nodes on US. 


\begin{tabular}{|l|l|l|}
\hline & MRI reader 1 (n=46) & MRI reader 2 (n=46) \\
\hline \multirow{2}{*}{ Sensitivity } & $84.4 \%(27 / 32)$ & $84.4 \%(27 / 32)$ \\
& {$[67.2-94.7]$} & {$[67.2-94.7]$} \\
\hline \multirow{2}{*}{ Specificity } & $35.7 \%(5 / 14)$ & $42.9 \%(6 / 14)$ \\
& {$[12.8-64.9]$} & {$[17.7-71.1]$} \\
\hline \multirow{2}{*}{ PPV } & $75.0 \%(27 / 36)$ & $77.1 \%(27 / 35)$ \\
& {$[57.8-87.9]$} & {$[59.9-89.6]$} \\
\hline \multirow{2}{*}{ NPV } & $50.0 \%(5 / 10)$ & $54.5 \%(6 / 11)$ \\
& {$[18.7-81.3]$} & {$[23.4-83.3]$} \\
\hline \multirow{2}{*}{ Accuracy } & $69.6 \%(32 / 46)$ & $71.7 \%(33 / 46)$ \\
& {$[54.2-82.3]$} & {$[56.5-84.0]$} \\
\hline
\end{tabular}

Table 3. Diagnostic performance of standard breast MRI for differentiation between pN1 and pN2-3 if ultrasound showed 1-3 suspicious axillary lymph nodes. Abbreviations: PPV, positive predictive value; NPV, negative predictive value. Data in parenthesis are absolute numbers. Data in brackets are $95 \%$ confidence intervals.

A standard breast MRI is frequently added to the diagnostic workup of breast cancer patients for a more accurate display of tumour size or extent. Since breast MRI includes the complete FOV of the axillary region in 1 out of 3 patients, it may provide the radiologist with the opportunity to evaluate axillary lymph node metasta$\operatorname{ses}^{36}$. Compared to the US, MRI has the advantage to visualise deeper-lying lymph nodes, the ability to assess the contralateral axilla at the same time, and lack of operator dependence ${ }^{37}$. An additional breast MRI in clinically node-positive patients has not been studied before. Hyun et al. reported that by adding breast MRI to negative cases of US and US-guided biopsy, pN2-3 was excluded in $98.0 \%$ of the patients ${ }^{38}$. In our study, we added a breast MRI to positive cases of US-guided biopsy. We showed that an additional breast MRI in clinically node-positive patients excluded pN2-3 in $75.7-77.8 \%$ of the patients. However, $38.5-41.7 \%$ of the patients were incorrectly classified as N2-3 axillary lymph node disease on breast MRI.

Since US and breast MRI are of limited value in clinically node-positive patients for differentiation between pN1 and pN2-3, the focus should perhaps shift to other imaging modalities. Other promising non-invasive imaging modalities are being investigated for the assessment of axillary lymph node metastases, such as PET/CT and PET/MRI. In the meta-analysis of Liang et al., the diagnostic performance of MRI and PET/CT was compared for the assessment of axillary lymph node metastases ${ }^{39}$. They showed that MRI (94.0\%) has a higher pooled area under the ROC curve compared to PET/CT (88.0\%) for diagnosing axillary lymph node metastases ${ }^{39}$. With the introduction of hybrid PET/MRI systems, the diagnostic advantages of MRI and PET are combined. Melsaether et al. showed that PET/MRI improves the diagnostic performance of axillary lymph node metastases detection from $88.0 \%$ to $100 \%$ compared to PET/CT ${ }^{40}$. In the Nijnatten et al. study, dedicated axillary PET/MRI was compared to other imaging modalities (i.e., US, MRI, and PET/CT $)^{41}$. PET/MRI resulted in a nodal status change of 40.0-75.0\% compared to US and MRI, respectively, and a nodal status change of $22.0 \%$ compared to PET/CT ${ }^{41}$. However, not all studies on PET/CT and PET/MRI take into account the number of lymph node metastases.

In the present study, size and morphology were used for the assessment of axillary lymph node metastases on the unenhanced T2-weighted MR sequence. The unenhanced MR imaging seems to be one of the most promising sequences for axillary lymph node metastases assessment due to the anatomical characterisation of the axillary lymph nodes ${ }^{42,43}$. It has been previously shown that the use of a dedicated axilla coil improves the accuracy of axillary lymph node staging ${ }^{44-47}$. However, it was chosen to use the size and morphology on the unenhanced T2-weighted breast MR sequence to facilitate possible implementation in other hospitals.

This study has certain limitations. The analyses were based on a small group of patients. This might have caused clinically meaningful differences in diagnostic performance to go undetected. However, the differentiation between pN1 and pN2-3 has not been studied before in clinically node-positive patients. Sample size expansion is not possible given that nowadays almost every clinically node-positive patient receives NST. In addition, the diagnostic performance parameters were based on a patient-by-patient level instead of a node-by-node level. All patients were treated with ALND, but the precise correlation between the visualised suspicious lymph nodes on US and breast MRI and the pathological results were not available, resulting in no node-by-node analysis. Further, different MRI scanners with varying protocols were used in the two medical centres. Given the small sample size in this study, a comparison between MR protocols was not possible. However, all MRI systems and protocols conform to the guidelines of the European Society of Breast Imaging. Finally, in this study, a dedicated axillary MR protocol was not available. A dedicated axilla coil could have improved the diagnostic performance parameters of MRI. Although, all MRI exams were pre-screened for a complete FOV of the axillary region.

\section{Conclusion}

In clinically node-positive breast cancer patients, the diagnostic performance of preoperative axillary US and standard breast MRI is comparable and both imaging modalities are unable to accurately differentiate between $\mathrm{pN} 1$ and $\mathrm{pN} 2-3$. An additional preoperative standard breast MRI can correctly diagnose pN2-3 in only 50.0$54.5 \%$ of the patients when US showed 1-3 suspicious axillary lymph nodes. Based on this study, there is no added clinical value of preoperative standard breast MRI for differentiation between pN1 and pN2-3 in patients with positive axillary US. 


\section{Data availability}

The datasets generated during and/or analysed during the current study are available from the corresponding author on reasonable request.

Received: 13 May 2019; Accepted: 7 November 2019; Published online: 25 November 2019

\section{References}

1. Banerjee, M., George, J., Song, E. Y., Roy, A. \& Hryniuk, W. Tree-based model for breast cancer prognostication. Journal of Clinical Oncology 22, 2567-2575, https://doi.org/10.1200/Jco.2004.11.141 (2004).

2. Senkus, E. et al. Primary breast cancer: ESMO Clinical Practice Guidelines for diagnosis, treatment and follow-up. Ann Oncol 26(Suppl 5), v8-30, https://doi.org/10.1093/annonc/mdv298 (2015).

3. CBO Kwaliteitsinstituut voor de Gezondheidszorg. Vereniging van Integrale Kankercentra. Guideline 'Treatment of breast cancer' (Richtlijn 'Behandeling van het Mammacarcinoom'). (2012).

4. Harnett, A., Smallwood, J., Titshall, V., Champion, A. \& Guideline Development, G. Diagnosis and treatment of early breast cancer, including locally advanced disease-summary of NICE guidance. BMJ 338, b438, https://doi.org/10.1136/bmj.b438 (2009).

5. Senkus, E. et al. Primary breast cancer: ESMO Clinical Practice Guidelines for diagnosis, treatment and follow-up. Ann Oncol 24(Suppl 6), vi7-23, https://doi.org/10.1093/annonc/mdt284 (2013).

6. Caudle, A. S. et al. Improved Axillary Evaluation Following Neoadjuvant Therapy for Patients With Node-Positive Breast Cancer Using Selective Evaluation of Clipped Nodes: Implementation of Targeted Axillary Dissection. Journal of Clinical Oncology 34, 1072-+, https://doi.org/10.1200/Jco.2015.64.0094 (2016).

7. Donker, M. et al. Marking Axillary Lymph Nodes With Radioactive Iodine Seeds for Axillary Staging After Neoadjuvant Systemic Treatment in Breast Cancer Patients The MARI Procedure. Annals of Surgery 261, 378-382, https://doi.org/10.1097/ Sla.0000000000000558 (2015).

8. van Nijnatten, T. J. A. et al. A Novel Less-invasive Approach for Axillary Staging After Neoadjuvant Chemotherapy in Patients With Axillary Node-positive Breast Cancer by Combining Radioactive Iodine Seed Localization in the Axilla With the Sentinel Node Procedure (RISAS): A Dutch Prospective Multicenter Validation Study. Clin Breast Cancer 17, 399-402, https://doi.org/10.1016/j. clbc.2017.04.006 (2017).

9. Crane-Okada, R., Wascher, R. A., Elashoff, D. \& Giuliano, A. E. Long-term morbidity of sentinel node biopsy versus complete axillary dissection for unilateral breast cancer. Annals of Surgical Oncology 15, 1996-2005, https://doi.org/10.1245/s10434-0089909-y (2008).

10. van Nijnatten, T. J. et al. The diagnostic performance of sentinel lymph node biopsy in pathologically confirmed node positive breast cancer patients after neoadjuvant systemic therapy: A systematic review and meta-analysis. Eur J Surg Oncol 41, 1278-1287, https:// doi.org/10.1016/j.ejso.2015.07.020 (2015).

11. Donker, M. et al. Radiotherapy or surgery of the axilla after a positive sentinel node in breast cancer (EORTC 10981-22023 AMAROS): a randomised, multicentre, open-label, phase 3 non-inferiority trial. Lancet Oncol 15, 1303-1310, https://doi. org/10.1016/S1470-2045(14)70460-7 (2014).

12. Giuliano, A. E. et al. Locoregional Recurrence After Sentinel Lymph Node Dissection With or Without Axillary Dissection in Patients With Sentinel Lymph Node Metastases Long-term Follow-up From the American College of Surgeons Oncology Group (Alliance) ACOSOG Z0011 Randomized Trial. Annals of Surgery 264, 413-420, https://doi.org/10.1097/Sla.0000000000001863 (2016).

13. Galimberti, V. et al. Axillary dissection versus no axillary dissection in patients with sentinel-node micrometastases (IBCSG 23-01): a phase 3 randomised controlled trial. Lancet Oncol 14, 297-305, https://doi.org/10.1016/S1470-2045(13)70035-4 (2013).

14. Arslan, G., Altintoprak, K. M., Yirgin, I. K., Atasoy, M. M. \& Celik, L. Diagnostic accuracy of metastatic axillary lymph nodes in breast MRI. Springerplus 5, 735, https://doi.org/10.1186/s40064-016-2419-7 (2016).

15. Kuijs, V. J. et al. The role of MRI in axillary lymph node imaging in breast cancer patients: a systematic review. Insights Imaging 6 , 203-215, https://doi.org/10.1007/s13244-015-0404-2 (2015).

16. Hyun, S. J., Kim, E. K., Moon, H. J., Yoon, J. H. \& Kim, M. J. Preoperative axillary lymph node evaluation in breast cancer patients by breast magnetic resonance imaging (MRI): Can breast MRI exclude advanced nodal disease? Eur Radiol 26, 3865-3873, https://doi. org/10.1007/s00330-016-4235-4 (2016).

17. Neal, C. H., Daly, C. P., Nees, A. V. \& Helvie, M. A. Can preoperative axillary US help exclude N2 and N3 metastatic breast cancer? Radiology 257, 335-341, https://doi.org/10.1148/radiol.10100296 (2010).

18. Park, S. H. et al. Impact of preoperative ultrasonography and fine-needle aspiration of axillary lymph nodes on surgical management of primary breast cancer. Ann Surg Oncol 18, 738-744, https://doi.org/10.1245/s10434-010-1347-y (2011).

19. Yang, W. T., Chang, J. \& Metreweli, C. Patients with breast cancer: differences in color Doppler flow and gray-scale US features of benign and malignant axillary lymph nodes. Radiology 215, 568-573, https://doi.org/10.1148/radiology.215.2.r00ap20568 (2000).

20. van Roozendaal, L. M. et al. Clinically node negative breast cancer patients undergoing breast conserving therapy, sentinel lymph node procedure versus follow-up: a Dutch randomized controlled multicentre trial (BOOG 2013-08). BMC Cancer 17, 459, https:// doi.org/10.1186/s12885-017-3443-x (2017).

21. Deurloo, E. E. et al. Reduction in the number of sentinel lymph node procedures by preoperative ultrasonography of the axilla in breast cancer. Eur J Cancer 39, 1068-1073, https://doi.org/10.1016/s0959-8049(02)00748-7 (2003).

22. Baltzer, P. A. et al. Application of MR mammography beyond local staging: is there a potential to accurately assess axillary lymph nodes? evaluation of an extended protocol in an initial prospective study. AJR Am J Roentgenol 196, W641-647, https://doi. org/10.2214/AJR.10.4889 (2011).

23. Farshchian, N. et al. Diagnostic value of chemical shift artifact in distinguishing benign lymphadenopathy. Eur J Radiol 80, 594-597, https://doi.org/10.1016/j.ejrad.2010.10.005 (2011).

24. Edge, S. B. \& Compton, C. C. The American Joint Committee on Cancer: the 7th edition of the AJCC cancer staging manual and the future of TNM. Ann Surg Oncol 17, 1471-1474, https://doi.org/10.1245/s10434-010-0985-4 (2010).

25. Leisenring, W., Alonzo, T. \& Pepe, M. S. Comparisons of predictive values of binary medical diagnostic tests for paired designs. Biometrics 56, 345-351 (2000).

26. Altman, D. G. Practical statistics for medical research. 1st edn, (Chapman and Hall, 1991).

27. National Institutes of Health Consensus Development, P. National Institutes of Health Consensus Development Conference statement: adjuvant therapy for breast cancer, November 1-3, 2000. J Natl Cancer Inst Monogr, 5-15 (2001).

28. Poortmans, P. M. et al. Internal Mammary and Medial Supraclavicular Irradiation in Breast Cancer. N Engl J Med 373, 317-327, https://doi.org/10.1056/NEJMoa1415369 (2015).

29. Whelan, T. J., Olivotto, I. A. \& Levine, M. N. Regional Nodal Irradiation in Early-Stage Breast Cancer. N Engl J Med 373, 1878-1879, https://doi.org/10.1056/NEJMc1510505 (2015).

30. Jagsi, R. et al. Complications After Mastectomy and Immediate Breast Reconstruction for Breast Cancer: A Claims-Based Analysis. Ann Surg 263, 219-227, https://doi.org/10.1097/SLA.0000000000001177 (2016). 
31. Kronowitz, S. J. Current status of implant-based breast reconstruction in patients receiving postmastectomy radiation therapy. Plast Reconstr Surg 130, 513e-523e, https://doi.org/10.1097/PRS.0b013e318262f059 (2012).

32. Lee, B. T. et al. Postmastectomy radiation therapy and breast reconstruction: an analysis of complications and patient satisfaction. Ann Plast Surg 64, 679-683, https://doi.org/10.1097/SAP.0b013e3181db7585 (2010).

33. Veronesi, P. et al. Immediate breast reconstruction after mastectomy. Breast 20(Suppl 3), S104-107, https://doi.org/10.1016/S09609776(11)70305-8 (2011).

34. Abe, H. et al. Axillary lymph nodes suspicious for breast cancer metastasis: sampling with US-guided 14-gauge core-needle biopsy-clinical experience in 100 patients. Radiology 250, 41-49, https://doi.org/10.1148/radiol.2493071483 (2009).

35. Schipper, R. J. et al. Axillary ultrasound for preoperative nodal staging in breast cancer patients: is it of added value? Breast 22, 1108-1113, https://doi.org/10.1016/j.breast.2013.09.002 (2013).

36. van Nijnatten, T. J. A. et al. Routine use of standard breast MRI compared to axillary ultrasound for differentiating between no, limited and advanced axillary nodal disease in newly diagnosed breast cancer patients. Eur J Radiol 85, 2288-2294, https://doi. org/10.1016/j.ejrad.2016.10.030 (2016).

37. Abe, H. et al. Accuracy of axillary lymph node staging in breast cancer patients: an observer-performance study comparison of MRI and ultrasound. Acad Radiol 20, 1399-1404, https://doi.org/10.1016/j.acra.2013.08.003 (2013).

38. Hyun, S. J., Kim, E. K., Yoon, J. H., Moon, H. J. \& Kim, M. J. Adding MRI to ultrasound and ultrasound-guided fine-needle aspiration reduces the false-negative rate of axillary lymph node metastasis diagnosis in breast cancer patients. Clin Radiol 70, 716-722, https:// doi.org/10.1016/j.crad.2015.03.004 (2015).

39. Liang, X. et al. MRI and FDG-PET/CT based assessment of axillary lymph node metastasis in early breast cancer: a meta-analysis. Clin Radiol 72, 295-301, https://doi.org/10.1016/j.crad.2016.12.001 (2017).

40. Melsaether, A. N. et al. Comparison of Whole-Body (18)F FDG PET/MR Imaging and Whole-Body (18)F FDG PET/CT in Terms of Lesion Detection and Radiation Dose in Patients with Breast Cancer. Radiology 281, 193-202, https://doi.org/10.1148/ radiol.2016151155 (2016).

41. van Nijnatten, T. J. A. et al. Diagnostic performance of gadofosveset-enhanced axillary MRI for nodal (re)staging in breast cancer patients: results of a validation study. Clin Radiol 73, 168-175, https://doi.org/10.1016/j.crad.2017.09.005 (2018).

42. Scaranelo, A. M., Eiada, R., Jacks, L. M., Kulkarni, S. R. \& Crystal, P. Accuracy of unenhanced MR imaging in the detection of axillary lymph node metastasis: study of reproducibility and reliability. Radiology 262, 425-434, https://doi.org/10.1148/ radiol.11110639 (2012).

43. Schipper, R. J. et al. Diagnostic Performance of Dedicated Axillary T2- and Diffusion-weighted MR Imaging for Nodal Staging in Breast Cancer. Radiology 275, 345-355, https://doi.org/10.1148/radiol.14141167 (2015).

44. Kvistad, K. A. et al. Axillary lymph node metastases in breast cancer: preoperative detection with dynamic contrast-enhanced MRI. European Radiology 10, 1464-1471, https://doi.org/10.1007/s003300000370 (2000).

45. Li, C. M., Meng, S., Yang, X. H., Wang, J. \& Hu, J. N. The Value of T2*in Differentiating Metastatic from Benign Axillary Lymph Nodes in Patients with Breast Cancer - A Preliminary In Vivo Study. Plos One, 9, doi:ARTN e8403810.1371/journal.pone.0084038 (2014).

46. Luciani, A. et al. Simultaneous bilateral breast and high-resolution axillary MRI of patients with breast cancer: preliminary results. AJR Am J Roentgenol 182, 1059-1067, https://doi.org/10.2214/ajr.182.4.1821059 (2004).

47. Yoshimura, G. et al. Evaluation of Axillary Lymph Node Status in Breast Cancer with MRI. Breast Cancer 6, 249-258 (1999).

Author contributions

Study concepts: S.S., T.v.N., R.J.S., Study design: S.S., T.v.N. Data acquisition: S.S., T.v.N., R.J.S. Quality control of data and algorithms: S.S. Statistical analysis: S.S, S.v.K. Manuscript preparation: S.S, T.v.N., R.J.S. Manuscript editing: S.S., T.v.N., H.v.B., M.P., A.M.B., E.H., S.v.K., R.J.S., M.L., M.S. Manuscript review: S.S., T.v.N., H.v.B., M.P., A.M.B., E.H., S.v.K., R.J.S., M.L., M.S.

\section{Competing interests}

S. Samiei received a salary from Alpe d'Huzes Foundation (Dutch Cancer Society; grant number: UM 20136229).

\section{Additional information}

Supplementary information is available for this paper at https://doi.org/10.1038/s41598-019-54017-0.

Correspondence and requests for materials should be addressed to S.S.

Reprints and permissions information is available at www.nature.com/reprints.

Publisher's note Springer Nature remains neutral with regard to jurisdictional claims in published maps and institutional affiliations.

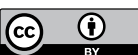

Open Access This article is licensed under a Creative Commons Attribution 4.0 International License, which permits use, sharing, adaptation, distribution and reproduction in any medium or format, as long as you give appropriate credit to the original author(s) and the source, provide a link to the Creative Commons license, and indicate if changes were made. The images or other third party material in this article are included in the article's Creative Commons license, unless indicated otherwise in a credit line to the material. If material is not included in the article's Creative Commons license and your intended use is not permitted by statutory regulation or exceeds the permitted use, you will need to obtain permission directly from the copyright holder. To view a copy of this license, visit http://creativecommons.org/licenses/by/4.0/.

(C) The Author(s) 2019 